Didáctica. Lengua y literatura

ISSN: 1130-0531

http://dx.doi.org/10.5209/DIDA.61958

\title{
El tratamiento del Marketing en los libros de texto de inglés para los negocios
}

\author{
Paloma López Zurita ${ }^{1}$
}

Recibido: 25 de noviembre de 2016 / Aceptado: 22 de junio de 2017

\begin{abstract}
Resumen. La importancia del Marketing en el ámbito económico ha sufrido una fuerte evolución en las últimas décadas, expandiéndose internacionalmente a través del inglés como lingua franca. Este desarrollo debería tener una repercusión directa en la composición de los libros para la enseñanza del inglés de los negocios dirigidos a estudiantes universitarios, que cada vez más necesitan ampliar sus conocimientos en esta disciplina. Por ello, el objetivo del presente estudio es comprobar si la generación de estas nuevas necesidades se ha visto reflejada en esos libros de texto en consonancia con el desarrollo del Marketing, desde la década de los 80 a la actualidad, a través del análisis de contenido de 123 ejemplares publicados en las fechas mencionadas. Los resultados muestran el grado de inclusión diacrónica de contenidos específicos de Marketing en las diferentes décadas, el tipo de tratamiento recibido y el nivel de los libros de texto en los que se desarrolla, así como la evolución del propio concepto Marketing en los mismos.
\end{abstract}

Palabras clave: IFE; Marketing; ILE; libros de texto.

\section{[en] The Treatment of Marketing in Business English Textbooks}

\begin{abstract}
The relevance of Marketing in the business world has undergone a significant evolution in recent decades, expanding internationally in English as a lingua franca. This development should have a direct impact in the content of Business English textbooks aimed at university students, who increasingly need to expand their knowledge of this discipline. Therefore, the main aim of this study is to check whether the generation of these new needs has had a reflection in those textbooks in accordance with the growth of Marketing, from the 80s up to now, through the content analysis of 123 textbooks published in the aforementioned dates. Results show the degree of diachronic inclusion of specific Marketing contents in different decades, the type of treatment received and the level of English of the textbooks which develop those contents, as well as the changes that the concept Marketing has evolved in them.
\end{abstract} Keywords: ESP; marketing; EFL; textbooks.

\section{[fr] Le traitement du Marketing dans les livres d'anglais pour les affaires}

Résumé. L'importance du marketing dans l'environnement économique a évolué fortement dans les dernières décennies, en s'étendant internationalement à travers l'anglais comme lingua franca. Ce développement devrait avoir une incidence directe sur le contenu des livres pour l'enseignement de l'anglais des affaires dirigés aux étudiants universitaires, qui ont besoin d'approfondir leurs connaissances de cette matière. C'est pourquoi le but de cette étude est celui de vérifier si la naissance de ces nouveaux besoins s'est reflétée dans ces livres de classe en rapport au développement du marketing, depuis des

1 Facultad de Ciencias Sociales y de la Comunicación. Universidad de Cádiz (España)

E-mail:paloma.lopez@uca.es 
80 jusqu'à ce moment, à travers l'analyse du contenu de 123 exemplaires parus à ces dates. Les résultats nous montrent le degré d'inclusion diachronique des contenus spécifiques du marketing dans ces décennies, le type de traitement reçu et le niveau des livres de classe dans lesquels se développe, ainsi que l'évolution du concept Marketing dans ces livres.

Mots-clés: Anglais sur Objectifs Spécifiques (AOS) ; Marketing ; ALE ; livres de classe.

Sumario. 1. Introducción. 2. Objetivos y metodología. 3. Marco teórico. 4. Resultados. 4.1. Evolución del tratamiento de Marketing en los libros de texto de Business English. 4.2. Nivel de los ejemplares. 4.3. Especialización progresiva de los contenidos. 5. Conclusiones. 6. Bibliografía. 7. Anexo: Libros de texto analizados (orden alfabético).

Cómo citar: López Zurita, P. (2018) El tratamiento del Marketing en los libros de texto de inglés para los negocios, Didáctica. Lengua y literatura, 30, 117-135.

\section{Introducción}

La expansión del alcance del Marketing en el mundo de los negocios es cada día más notorio como reflejo de los cambios experimentados por la sociedad y la economía, donde los aspectos comerciales, influidos sobre todo por el desarrollo de las nuevas tecnologías y la imparable evolución de internet, lo convierten en una herramienta casi indispensable para la realización de cualquier actividad mercantil: "A estas alturas nadie -o casi nadie- discute la importancia del Marketing en la vida y en el éxito de las empresas, en el conjunto de la economía de un país y en la internacionalización de las relaciones económicas." (Martínez, 2008, 10).

Su influjo se difunde en todos los ámbitos, creándose modalidades específicas según el campo de acción y las estrategias de Marketing empleadas; se habla de Marketing turístico (Hurtado, 2016), político (Martín, 2002), cultural (Lee y Littrell, 2006), económico (Kotler y Armstrong, 2003; Santesmases, 2014), etc. A veces, incluso se supera el matiz mercantil inicial con metas que van más allá de la comercialización de un producto, un servicio o incluso la difusión de una ideología, llegando a tener otros objetivos, como la mejora de la imagen de una empresa, producto, marca, etc., a través del Marketing reputacional (Pérez y Rodríguez del Bosque, 2014), la fidelización, no sólo del cliente sino también de los propios empleados, a través del Marketing corporativo o motivacional (Vieira, Higuchi, Schneider y Soares, 2013), o la concienciación sobre temas diversos, como el Marketing social (Zenone, 2006) y el ecológico, también denominado Marketing verde o eco-Marketing (Vicente, 2014), entre otros.

Ante todas estas denominaciones, el Diccionario de la Real Academia Española (2014) recoge el término "Marketing" como voz inglesa y nos remite a su equivalente español "Mercadotécnica". Es bajo esta entrada donde se define como "1. f. Econ. Conjunto de principios y prácticas que buscan el aumento del comercio, especialmente de la demanda. 2. f. Econ. Estudio de la mercadotecnia." Pese a las recomendaciones de uso de este vocablo por parte de la Academia y a la propuesta de su adaptación como "márquetin" por el Diccionario Panhispánico de Dudas (2005), en España el anglicismo crudo sigue siendo el más utilizado frente al anterior, a 
"mercadotecnia" e incluso frente a "mercadeo", ambos habituales en algunos países de Latinoamérica. Será pues, por el impacto de su expansión, el término que utilicemos en este artículo.

En este escenario, la importancia del inglés es esencial debido a la masificación global y al carácter internacional de un gran número de acciones de Marketing a través de Internet. Esta herramienta es utilizada no sólo por las empresas sino también por el propio usuario individual, que cada vez utiliza más las técnicas one to one. Se ha incrementado, pues, el uso del inglés aplicado al Marketing, con una terminología propia que se extiende incluso a los propios investigadores en el ámbito académico. Estos no sólo deben dominar un discurso preñado de neologismos en continua renovación que da respuestas a estas nuevas realidades, sino que también han de enfrentarse a unas características propias en la redacción de sus artículos especializados en esta disciplina (Blesa y Fortanet, 2003). Todo ello crea realidades, conceptos y estructuras dotadas de una terminología específica cada vez más novedosa y cambiante que el alumnado especializado en esta disciplina debe adquirir y que debe tenerse en cuenta en el proceso de enseñanza-aprendizaje del inglés aplicado a esta materia.

En el contexto universitario español, los estudios de Marketing no comenzaron a impartirse como Grado de manera independiente hasta la adaptación del sistema educativo al Espacio Europeo de Enseñanza Superior, que comenzó con las Declaraciones de la Sorbona y Bolonia en 1998 y1999, respectivamente, y se vio culminado en el curso 2010-2011. Antes de estas fechas, el Marketing se incluía bien como especialidad en Gestión Comercial en la anterior Diplomatura en Empresariales o bien se ofertaba como segundo ciclo de la Licenciatura en Técnicas e Investigación de Mercados. Sin embargo, fruto de la existencia de esas nuevas realidades que comentábamos anteriormente, se hizo necesario y conveniente la creación de nuevos estudios con nuevas competencias y contenidos, creándose un nuevo Grado en Marketing e Investigación de Mercados, bajo esta misma denominación u otras análogas.

\section{Objetivos y metodología}

Tanto en las titulaciones previas mencionadas anteriormente como en los actuales Grados de la rama de empresa, los planes de estudio suelen incluir asignaturas de inglés aplicado, bien al marco específico del Marketing, bien a un contexto empresarial más general del que este también forma parte. En este contexto, el objetivo del presente estudio es comprobar si la generación de estas nuevas necesidades ha tenido o no un reflejo en los contenidos de los libros de texto para la enseñanza del inglés aplicado a la empresa y los negocios. En concreto, se analizará si los contenidos relativos al Marketing se han incrementado o no en estos manuales en consonancia con el aumento de la importancia de esta disciplina en el ámbito empresarial, social y económico.

Para lograr el objetivo anterior, se ha llevado a cabo un análisis de contenido de 123 libros destinados a la enseñanza del inglés aplicado a la empresa, los negocios y al propio Marketing, en su caso, publicados desde la década de los 80 hasta 2016. Dada la amplia tradición de los estudios mercantiles en la Universidad de Cádiz, que datan de más de un siglo (Guilloto, 1994), su biblioteca cuenta con un amplio catálogo bibliográfico sobre este tema, por lo se han extraído todos los ejemplares 
existentes en la misma utilizados por el profesorado en algún momento del periodo seleccionado.

En este estudio cualitativo hemos distinguido dos partes que se desarrollan paralelamente: Una primera que responde a las siguientes preguntas y a las implicaciones que sus resultados conllevan: 1. ¿a qué décadas corresponden cada uno de los ejemplares analizados?; 2. ¿cuántos incluyen alguna referencia al Marketing entre sus contenidos?; 3. ¿en esos casos, qué tratamiento se le ha dado? es decir, si se incluye solo a través de actividades puntuales, si incluye unidades específicas sobre Marketing o si se trata de ejemplares exclusivos de esta disciplina; 4. ¿cuál es el nivel de inglés de cada ejemplar de acuerdo con el punto anterior?; y, finalmente, 5. ¿cuál ha sido la evolución de este tratamiento por décadas, desde los años 80 al periodo a partir del año 2000? En segundo lugar, se ha analizado si los contenidos hallados se ciñen a conceptos tradicionales de compra-venta o se incorporan modelos más innovadores hasta llegar al concepto holístico del Marketing actual. De ahí, intentamos comprobar cómo ha ido evolucionando el tratamiento del Marketing, desde centrarse tan solo en actividades de gestión comercial o ventas hasta incluir temas mucho más especializados como son el servicio y atención al cliente, el Marketing directo, estudios de merchandising, el diseño del plan de Marketing, Marketing mix, análisis de la competencia, plan de fidelización de clientes, previsión de ventas y gestión de stocks, planificación, ejecución y análisis de encuestas sobre satisfacción de los clientes, las nuevas tecnologías como instrumentos de Marketing, etc., contenidos todos incluidos en los nuevos Grados de Marketing que comentábamos en la introducción al presente artículo.

\section{Marco teórico}

En las últimas décadas, el Business English ha cobrado una importancia considerable, reflejada en la abundancia de libros y cursos para la enseñanza del inglés con ese propósito específico. Sin embargo, a veces parece que la respuesta editorial ante la relevancia adquirida por el Marketing en los últimos años no ha sido la misma. Aunque hay autores que opinan que la tendencia es inversa en la mayoría de los nuevos campos de especialidad, nos parece que esto no se refleja así en el ámbito del Marketing:

[...] La respuesta ha venido de la mano del enorme impulso que autores, editoriales y mercado están dando en conjunto al desarrollo de las lenguas de especialidad. Sin embargo, esta afirmación no quiere decir que la progresión se esté produciendo de manera equilibrada y con la misma velocidad en el campo de la investigación teórica, práctica y metodológica de una parte, y la elaboración de materiales didácticos por otra. Precisamente, el desajuste mayor en este campo reside en el desfase creciente en detrimento de la investigación teórico-práctica rigurosa y en favor de la elaboración de materiales para la enseñanza. Esta situación es atípica, porque normalmente la investigación teórica debería preceder las aplicaciones prácticas. (Felices, 2005, 81)

En el caso que nos ocupa, coincidimos con Felices (2005) en que las investigaciones teórico-prácticas sobre esta lengua de especialidad todavía se encuentran 
en un estado incipiente, añadiríamos que casi inexistente. Sin embargo, tampoco podemos afirmar que exista desfase entre este hecho y la elaboración de materiales para la enseñanza, puesto que esta sigue siendo también escasa, ya que de la muestra analizada solo 6 de los 123 libros de texto se dedican al ámbito del inglés aplicado al Marketing en exclusividad.

Entre los escasos ejemplos de investigación teórica, práctica y metodológica existentes sobre la importancia de la lengua inglesa en Marketing, se encuentra Hyland (1999), que incorpora a su análisis sobre el papel de los libros de texto en la adquisición de una disciplina especializada un corpus de 21 libros donde incluye el Marketing junto a otras materias totalmente dispares como la Microbiología y la Lingüistica Aplicada; Palmer (1999), que roza el Marketing de soslayo al analizar el uso de anuncios en la clase de Business English; Bobáková (2010), que realiza un estudio sobre los anglicismos en este ámbito, en los idiomas alemán y checo; Barsanti (2000), que aporta un estudio léxico en esta disciplina, junto a la Publicidad y la Comunicación, en francés y alemán, o Mendoza, Mendoza-Ponce y Mendoza (2015), que aportan un enfoque metodológico común entre ambas en el contexto universitario ecuatoriano. Como sucede también en algunos de los ejemplares analizados y se verá más adelante, el Marketing suele confundirse con la publicidad y en ese campo particular sí hay más avances teóricos (Durán, 2000 y 2002; Gea, 2005; Luján, 2010; Venegas y Ocaña, 2016), etc.

En cuanto al análisis del contenido de libros de texto en general, los existentes suelen tener un matiz pedagógico y metodológico enfocado principalmente a alumnos de primaria o secundaria (Jiménez, 2003; García, 2015; Braga y Belver, 2016) o, en este mismo contexto, al desarrollo de determinadas destrezas o capacidades lingüísticas, como la lectura (Puchmüller, Noriega y Fattori, 2010), la comprensión (González, 2015), la escritura (Pérez, Nodarse y Mons, 2013), etc. Pese a esta escasez en el marco teórico, la importancia de esta lengua en el ámbito de Marketing está contrastada por su fuerte presencia e influencia (Figueroa, 1999) y parece que la interrelación entre ambas disciplinas va cobrando cada vez más interés, aunque se deba a la docencia de una a través de la otra en el contexto de innovación educativa universitaria (Bravo, Jiménez y Pina, 2011; Núñez, Rey y Ceular, 2011).

Por todo ello, aunque en Internet proliferan los glosarios y los ejercicios gramaticales sobre temas puntuales de Marketing, la elaboración de materiales para su enseñanza a través de libros de texto tampoco ha experimentado el impetuoso desarrollo que sí se ha producido en la realidad económica actual. Sin embargo, sí se detecta una evolución en los contenidos de Marketing incluidos en los libros de Business English, como se verá más adelante.

\section{Resultados}

\subsection{Evolución del tratamiento de Marketing en los libros de texto de Business English}

Los 123 libros de texto sobre la enseñanza del inglés de los negocios analizados han sido publicados a partir de los años 80 hasta 2016 (Ver Anexo). El primero de ellos en esta cronología corresponde al año 1978, pero tratándose de una fecha tan tardía en la década, ha sido incluido junto a los de la década posterior. 


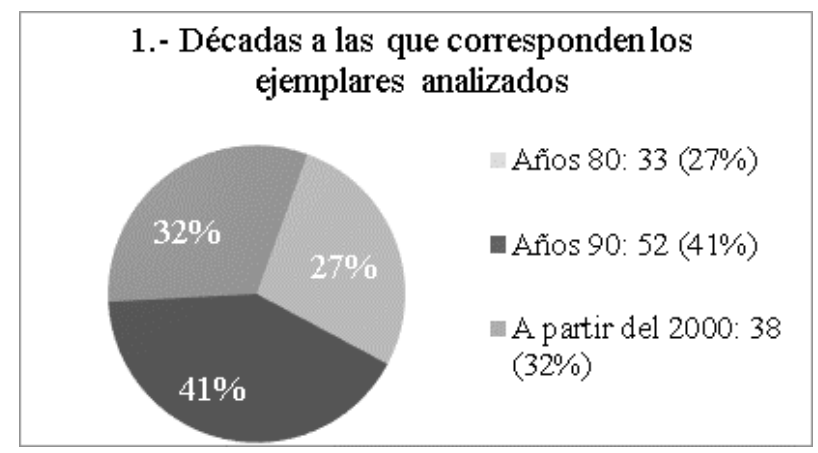

Gráfico 1. Décadas.

Como se observa en el gráfico anterior, 33 ejemplares corresponden a la década de los $80(27 \%), 52$ a los años $90(41 \%)$ y 38 fueron publicados a partir del año 2000 (32\%), con lo que se observa el gran auge que el inglés para los negocios adquirió en los años 90, corroborando lo ya apuntado por Bueno y Hernández (2002) en su panorama histórico sobre el desarrollo del Inglés para Fines Especificos (IFE): "Otra de las tendencias a la que ya se había hecho referencia es la del predominio de materiales y cursos destinados al inglés para negocios que ha relegado a un segundo plano la producción de materiales de Inglés para la ciencia y la tecnología cuando estos fueron los que en un inicio ocuparon un lugar preferente."

En cuanto a la presencia de contenidos referentes a Marketing en los ejemplares analizados, en 67 casos (54\%) no se incluye referencia alguna a esta disciplina, mientras que en 56 sí (46\%). Aun así, se observa que la mayoría de aquellos que lo incluyen le dan la importancia suficiente como para hacerlo a través de temas o unidades específicas completas, ya que estos suponen el 70\% del total (39 ejemplares), frente a un 30\% (17 ejemplares), que sólo lo tratan a través de actividades puntuales insertas en el marco de otros contenidos principales:

\begin{tabular}{|c|c|}
\hline \multirow{2}{*}{$\begin{array}{l}\text { 2.- Presencia de Marketing en los } \\
\text { ejemplares analizados } \\
\qquad \begin{array}{l}\text { No se incluye: } \\
67(54 \%)\end{array}\end{array}$} & $\begin{array}{l}\text { 3.- Tratamiento del Marketing en los } \\
\text { ejemplares analizados }\end{array}$ \\
\hline & $\begin{array}{l}\text { A través de } \\
\text { actividades } \\
\text { puntuales: } 17(30 \%)\end{array}$ \\
\hline $\begin{array}{l}\text { Incluye } \\
\text { Marketing: } 56 \\
(46 \%)\end{array}$ & $\begin{array}{l}\text { Incluyen unidades } \\
\text { especificas : } 39 \\
(70 \%)\end{array}$ \\
\hline
\end{tabular}

Gráficos 2 y 3. Presencia y Tratamiento del Marketing.

Para comprobar si la inclusión del Marketing ha sufrido o no una evolución temporal se han clasificado por décadas los 56 ejemplares que sí incluyen esta disciplina entre sus contenidos, obteniendo los siguientes datos: 

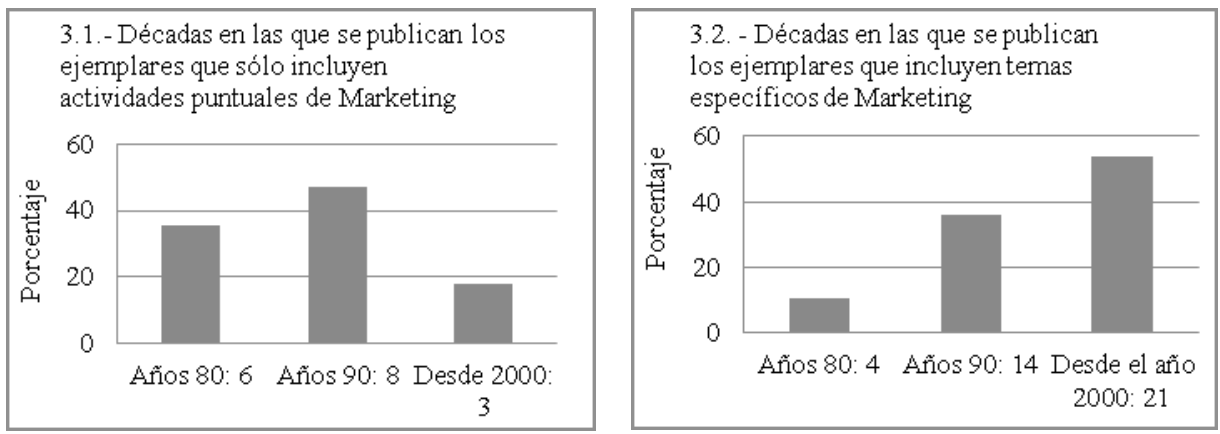

Gráficos 3.1 y 3.2. Tratamiento del Marketing por décadas.

Los gráficos anteriores son bastante significativos. Como se observa, en la década de los 80 la tendencia imperante era la de incluir sólo actividades puntuales, tendencia que se incrementó en los 90 y que disminuye notablemente a partir del 2000. Si comparamos estos datos con los reflejados en el gráfico 3.2, comprobamos cómo la tendencia es inversa y el número de ejemplares que incluyen temas completos dedicados al Marketing, dotándolo de más importancia, se incrementa notablemente. El estudio segmentado por décadas resulta aún más aclaratorio de estas tendencias inversas:

Años 80: En estos años, sólo el 30\% de los textos aludían al Marketing de alguna manera. La mayoría lo hacen a través de actividades puntuales, como refleja el gráfico 4.2. Sólo 4 de los 33 ejemplares analizados incluyen temas específicos y ninguno de ellos se dedica exclusivamente al Marketing.
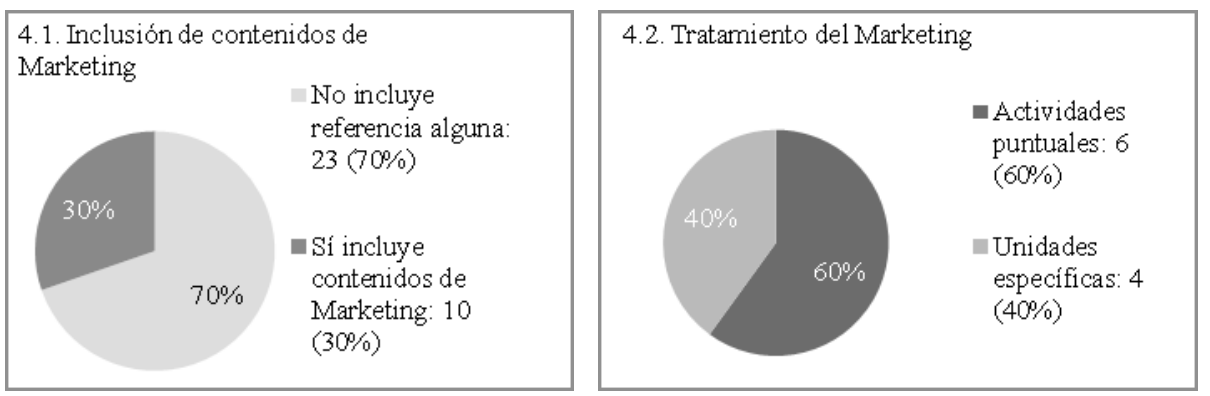

Gráfico 4.1. Inclusión de contenidos. Gráfico 4.2. Tratamiento del Marketing.

Década de los 80

Años 90: En los años 90 se comprueba cómo se incrementa el número de ejemplares que tienen en cuenta contenidos de Marketing, pasando del 30\% de la década anterior al $42 \%$ en esta. Su tratamiento también comienza a cambiar, pues ya son más los ejemplares que dedican unidades enteras específicas a esta disciplina en lugar de hacerlo sólo a través de actividades dispersas y puntuales, pasando del 12\% de la década anterior, al $55 \%$. Esta tendencia también se evidencia en que aparecen dos ejemplares especializados exclusivamente en Marketing. 

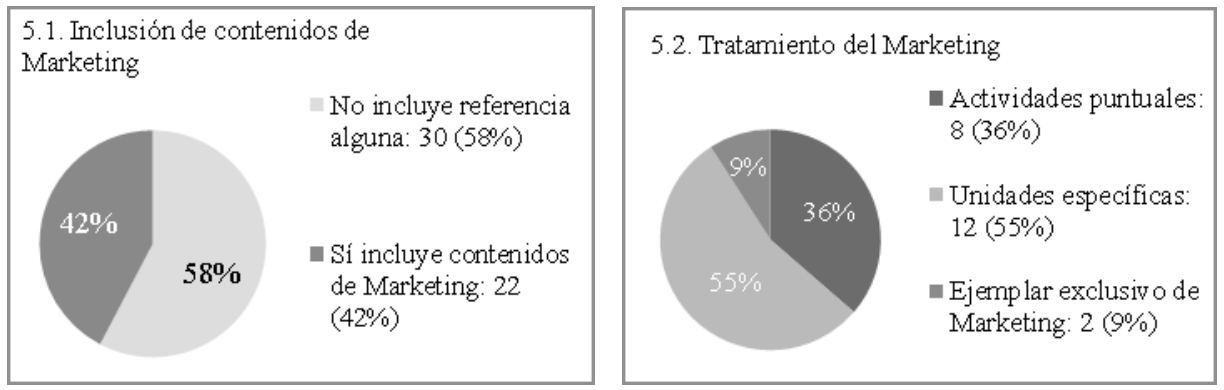

Gráfico 5.1. Inclusión de contenidos. Gráfico 5.2. Tratamiento del Marketing. Década de los 90.

A partir del año 2000: Con el nuevo siglo el auge del Marketing se refleja en el incremento de su presencia en los libros analizados: La mayoría lo incluye (63\%). Su tratamiento también es diferente, pues mientras que sólo en 3 casos se alude al tema puntualmente con alguna actividad (12\%), el mayor porcentaje recae en los ejemplares que le dedican unidades completas $(71 \%)$. La tendencia a la especialización también aumenta, con la aparición de 4 ejemplares dedicados exclusivamente a la especialización en esta disciplina.
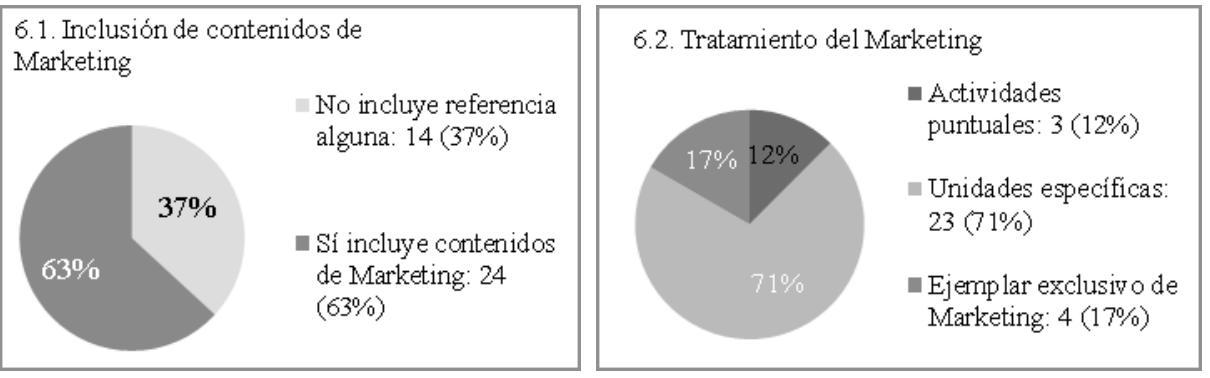

Gráfico 6.1. Inclusión de contenidos. Gráfico 6.2. Tratamiento del Marketing. A partir del año 2000.

\subsection{Nivel de los ejemplares ${ }^{2}$}

Ante los datos anteriores nos pareció también determinante conocer si esta tendencia al incremento del papel del Marketing, que parece haber quedado contrastada, se ejercía a todos los niveles o bien cuando el alumnado ya poseía cierto bagaje de inglés como para poder adentrarse en la adquisición de una terminología aún más específica dentro del marco de los negocios. Así pues, se llevó a cabo la clasificación de los distintos niveles de competencia idiomática de los ejemplares que sí incluían entre sus contenidos algún referente al Marketing. Por un lado, analizamos aquellos que lo hacían a través de actividades puntuales:

2 El nivel de los ejemplares se recoge tal y como aparece en los mismos. A modo de guía se podrían establecer las siguientes equivalencias con los niveles del Marco Común Europeo de Referencia para las Lenguas (MCER): beginner, A1; elementary, A2; lower intermediate to pre-intermediate, B1; intermediate to upper-intermediate, $\mathrm{B} 2 ;$ advanced, $\mathrm{C} 1$; proficiency, $\mathrm{C} 2$. 


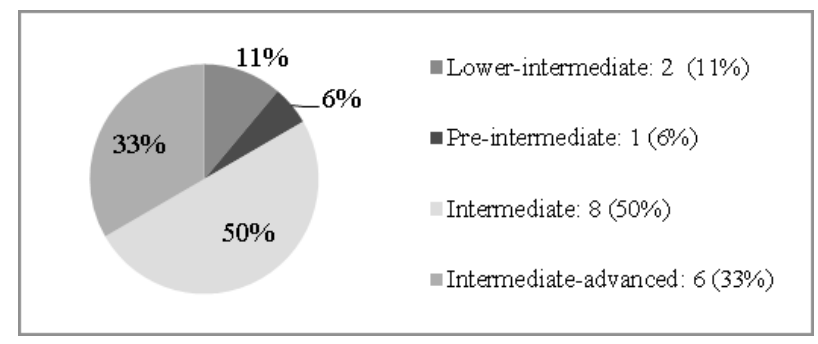

Gráfico 7. Nivel de los libros de texto que incluyen actividades puntuales.

Como se observa en el gráfico anterior, los niveles son heterogéneos, pero los porcentajes mayores corresponden con los niveles intermedio (50\%) o intermedio-avanzado $(33 \%)$.

Por otro lado, se segmentaron los niveles de aquellos ejemplares que incluían temas completos o constituían ellos mismos un ejemplar especializado en Marketing:

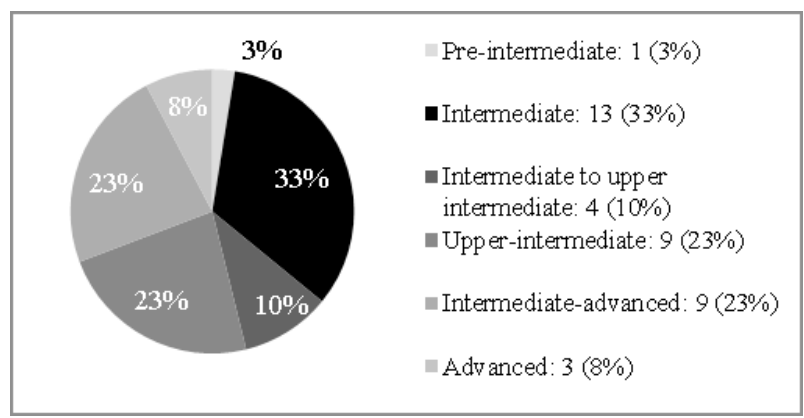

Gráfico 8. Nivel de los libros de texto con temas específicos de Marketing.

En este caso comprobamos que el nivel de los libros de texto se eleva: solo 1 ejemplar tiene un nivel inferior al intermedio y la mayoría de encuentra entre este nivel y el avanzado (89\%). Mientras que ya no aparece ningún texto con nivel inferior al pre-intermedio o inferior, sí aparecen, por el contrario, 3 casos de nivel avanzado.

Deducimos, pues, que al alumnado objetivo de los libros de texto con contenidos de inglés aplicado al Marketing se le presupone ya un cierto conocimiento de la lengua inglesa, de al menos un nivel intermedio. Cuanto más especializado es el ejemplar, mayores son los requisitos de conocer la lengua inglesa de base. Esto se corrobora por el hecho de que todos aquellos ejemplares específicos solo y exclusivamente de Marketing poseían un nivel que iba desde el intermedio-alto al avanzado, nunca inferior.

\subsection{Especialización progresiva de los contenidos}

Para comprobar si el enfoque dado al Marketing también había evolucionado o no, se llevó a cabo el análisis de los contenidos de aquellas actividades puntuales que lo abordaban inserto en otras unidades de diferente temática.

Inicialmente, la propia palabra "Marketing" no suele aparecer en los epígrafes de estas actividades, aunque su contenido sí esté relacionado con él. Es el caso de Vocabulary: Factors reflecting sales results (Fletcher y Hargreaves, 1983); Entering 
a new market; Expanding overseas o Facts and figures (White y Khidhayir, 1984); Sales call o Market share developments (Brieger et al., 1987); Sales review; Sales forecast; Market research (Brieger y Comfort, 1989) o, simplemente, Market (Wilberg y Lewis, 1991). Normalmente se trata de actividades relacionadas con el vocabulario utilizado en el proceso de ventas, de baja especificidad, y con la investigación de mercados, haciendo más hincapié en la descripción general de gráficos y tendencias, incluso en los números o cifras, que en la propia investigación de mercados en sí, ya que no se incluyen parámetros básicos como los tipos de investigación, las distintas metodologías, etc.

En aquellos casos en los que sí aparece el término "Marketing", los contenidos siguen ciñéndose, sin embargo, al campo casi exclusivo de las ventas y la investigación de mercados: Selling and Marketing (Rowlands, 1989); Marketing and sales (Comfort et al., 1990); Marketing research (Sandler y Stott, 1990); Initiating a market survey; Results of a market survey (Darragh y Rosset, 2008). Incluso en algunos ejemplares más actualizados, en los que se incluye el influjo de Internet y las nuevas tecnologías como estrategias novedosas, las actividades puntuales siguen refiriéndose a estos mismos conceptos y contenidos como eje central del Marketing. Es el caso de E-Commerce, Internet Marketing y Online shopping (Clarke, 2003).

En otras actividades se aprecia una mezcla difusa entre elementos de Marketing y de Publicidad, aun tratándose de conceptos distintos (Guiu, 2015). A veces se tratan conjuntamente, como en Marketing and promotion (McKellen, 1990) o en Describing products; Product features; Presenting a product; An advertising brief (Benn y Dimmet, 1992); otras, se incluyen contenidos de Marketing bajo el epígrafe de Publicidad, como en Brands o Advertising (Cotton et al., 2000) o viceversa, como en Products, markets and Marketing (Mascull, 1996), sin mencionar distinción alguna entre conceptos o la inclusión de la Publicidad como parte fundamental del Marketing.

En cuanto a los ejemplares que sí incluyen unidades específicas, esta misma tendencia reduccionista de minimizar el ámbito del Marketing al campo de las ventas y la investigación de mercados sigue manteniéndose en la década de los 80. Como ejemplos, A Market Research Questionnaire (Spiro, 1985); Advertising and Sales Promotion (Greenall, 1986); Market Research Survey (Danks y Cross, 1989).

En los 90 comienzan a introducirse nuevos conceptos, como el desarrollo del producto: Market Research and Product Development (Ryann y Richards. 1991); las diferencias terminológicas y conceptuales: Advertising vs Publicity vs Marketing vs Public Relations; Make vs Brand vs Trademark vs Logo vs Patent vs Copyright (Strutt, 1992); la influencia de la globalización, la gestión de marca o las técnicas de venta: Global Marketing; Brand Management; Sales Techniques (Powell, 1992); la estructura de mercado: Market Structure and Competition (Mackenzie, 1997) o el importante papel de la ética en el sector: Marketing Ethics (Cotton, 1998).

A partir del año 2000, estos contenidos comienzan a ampliarse, enfocando el Marketing desde una óptica más especializada, superando el básico concepto inicial del proceso compra-venta. La mayoría de los ejemplares incluye, como mínimo, una unidad especializada (Campbell, 2000; Tullis y Trappe, 2002; Naunton, 2009; Ceramella y Lee, 2010; Cotton, Falvey y Kent, 2012; Dubicka y O'Keeffe, 2016) que en muchos casos se complementa con otras unidades aún más específicas, con temas como los siguientes: 
- Atención al cliente: Tell Me What You Want (Naunton, 2000); Customer Satisfaction y Knowing Your Customer (Goodwin y Strurt, 2005).

- Merchandising o técnicas de venta: Promotional Tools (Mackenzie, 2002); The Art of Persuasion (Naunton, 2000).

- Estructura de Mercado: Marketing Structure and Competition (Mackenzie, 2002); Marketing and Market Orientation (Aspinall y Bethell, 2009); Markets and Competitors; Marketing and Market (Mascull, 2010).

- Tipos de Marketing y su publicidad: Product and Corporate Advertising (Tullis y Trappe, 2002); International Marketing (Cotton, 2006).

- Marketing mix: 4Ps; Products and Brands; Price; Place; Promotion (Aspinall y Bethell, 2009); The Marketing Mix (Nauton, 2009); The Four P's and Beyond (Mascull, 2010).

- Distinción entre Publicidad y Marketing: Marketing, Advertising and Sales (Campbell, 2000); Marketing; Advertising (Mackenzie, 2002); Brands and Branding (Goodwin y Strurt, 2005).

Son los ejemplares totalmente especializados en contenidos de Marketing los que realmente aportan una óptica completa para la enseñanza de inglés aplicado a esta materia (Comfort y Brieger, 1992a, 1992b; Renucci, 1997; Farral y Lindsley, 2008; Robinson, 2010; Gore, 2015) incluyendo unidades que recogen todo lo anterior, a las que se suman aspectos como plan de Marketing, análisis DAFO; estrategias y segmentación de mercados; logística y distribución, aspectos legales y éticos, el ciclo de vida de los productos, o nuevos tipos de Marketing, acuñados normalmente como anglicismos: Buzz Marketing, Street Marketing; Stealth Marketing, Motivation Marketing, etc.

El profesional en Marketing actual necesitará no solo un inglés aplicado a los negocios sino un inglés aplicado específicamente a esta materia. Esta necesidad se refleja en los contenidos de los libros analizados a través del aumento de la especialización de los mismos, como se ha detallado anteriormente. Así, los ejemplares más recientes incluyen desde los conceptos básicos del Marketing hasta las nuevas tendencias implementadas en internet. Un ejemplo es el caso de Farral y Lindsley (2008), Professional English in Use. Marketing, que comienza con conceptos tan tradicionales como el marketing mix o el SWOT analysis, para adentrarse en tipos y metodologías de investigación de mercados, el nuevo desarrollo de los productos y su ciclo de vida, el posicionamiento de marca, su imagen y valores, la creación del engagement, las nuevas estrategias del Marketing de $360^{\circ}$, etc. hasta la explicación de los últimos acrónimos en inglés más extendidos por el uso de internet, como $C P M$, CIM, B2B, B2C, C2C, DSS, EDP, etc.

\section{Conclusiones}

Aunque todavía de manera tímida, las editoriales dedicadas a la publicación de libros de texto para la enseñanza del inglés de los negocios parecen estar haciéndose eco de la relevancia que el Marketing ha adquirido en los últimos años en el marco de la propia economía, la sociedad y los estudios universitarios especializados. Como consecuencia, de no existir ningún ejemplar al inicio del periodo analizado (cuando 
tampoco existían estudios específicos e independientes de Marketing), en la última década sí se han publicado 4 libros de texto para hacer frente a esta necesidad (en consonancia con la creación del Grado en Marketing e Investigación de Mercados en el sistema educativo español).

Su tratamiento en los libros de inglés aplicado al mundo de los negocios también ha variado, mostrando esa misma tendencia ascendente hacia la incorporación de contenidos de Marketing: muestra un claro incremento gradual desde la década de $\operatorname{los} 80$, en donde su aparición era casi anecdótica, pasando a incluirlos a través de actividades puntuales dispersas basadas en el concepto básico de compra-venta y la investigación de mercados, insertando más tarde unidades completas especializadas, hasta hacer evidente la necesidad de ejemplares específicos que, aún de manera deficitaria, han comenzado a publicarse.

El escaso contenido de Marketing en las primeras décadas se podría justificar no solo por el aumento posterior de la relevancia de esta materia en general, sino también porque en aquellos momentos su estudio se encontraba inserto en ejemplares dirigidos a un alumnado de Administración y Dirección de Empresas o Empresariales, con unas necesidades conceptuales menores en este apartado, cuyo enfoque era básicamente comercial. Precisamente fue su auge internacional progresivo el que fue creando poco a poco las bases sobre las que se asentó la creación de los nuevos Grados en esta materia, dotándolo de una entidad propia e individual. Podríamos decir que ciertamente aquellos inicios cubrían las necesidades formativas y de empleabilidad de aquellos estudiantes de los 80, los 90 e inicios del 2000. Sin embargo, la transformación de la disciplina del Marketing en los últimos años ha sido tal que ha tenido que adaptar sus estrategias al poder casi omnímodo de la tecnología y su poderosa difusión, modificando sus técnicas hasta llegar a la relación directa con el usuario individual a través de internet, teniendo ante sí un abanico de opciones que se presumen casi ilimitadas en vista de la fuerte y rápida evolución que se está produciendo. Todos estos avances derivan en nuevos conceptos de Marketing que se desarrollan y expanden al mismo tiempo que la propia tecnología y que, como ya hemos comentado, comparten en este desarrollo la lingua franca en la que actualmente se suelen acuñar todos los nuevos términos que se generan: el inglés. Por esta razón, el inglés aplicado al Marketing, de ser un contenido específico en el marco de libros de texto para fines empresariales, presumiblemente prescindible dependiendo de la especialidad del alumnado, ha pasado a convertirse en una necesidad indispensable para entender las nuevas tendencias y su terminología que, como apuntábamos en el apartado anterior, se acuña y usa en el marco profesional normalmente a través de anglicismos, de los que ya comentamos algunos y a los que se pueden sumar Inbound Marketing, E-mail Marketing; Ambient Marketing, Co-branding, Blended Marketing, y un largo etc.

También se deduce que el alumnado debe ya haber adquirido la competencia lingüística necesaria para poder abordar temas de esta complejidad. Esto se refleja en que la mayoría de los ejemplares con mayor contenido en Marketing son de un nivel intermedio alto, no solo con estructuras lingüísticas más complejas, sino también con un léxico más avanzado y una terminología más específica, puesto que en esta evolución los contenidos especializados también van en aumento. Es en estos ejemplares donde la lengua se adquiere a través de los contenidos y donde tanto el alumnado como el profesorado han de poseer un conocimiento de ambas materias a un nivel más avanzado, donde el especialista en IFE debe mostrar un dominio mayor 
de la disciplina en cuestión, ajena a su formación inicial, para poder transmitirla. Como Fresno (2012) comenta, haciéndose eco de Robinson (1991) y Dudley-Evans y St. John (1998), ya clásicos en el campo de IFE:

No podemos afirmar que el profesor de IFE sea meramente un profesor, en realidad desempeña funciones de investigador, diseñador de cursos, creador de materiales, examinador, evaluador y, por supuesto, docente. Necesita poseer a) conocimientos teóricos y prácticos en lo referente a la propia lengua que imparte, en nuestro caso, inglés, b) conocimientos de la enseñanza de esa lengua y c) conocimientos del diseño del curso que va a impartir. Pero además requiere, a diferencia del profesor de Inglés General (IFG), poseer conocimientos del área profesional de los alumnos a los que imparte docencia, ya sea Ciencias económicas, Ciencias de la salud, Ingeniería Aeronáutica, Aviación, ingeniería Industrial [...] (Fresno, 2012, 110)

Cita que completaríamos añadiendo "o Marketing" a la lista anterior de ejemplos.

En este marco multitarea, esperemos que la tendencia por parte de las editoriales continúe en la progresión que hemos corroborado en este artículo y nos facilite la ya ardua y concurrente labor de enseñar inglés en esta disciplina con la publicación de más ejemplares especializados en Marketing, necesarios tanto en el modelo educativo universitario como en la sociedad actual. Suponemos que la escasa existencia de estos manuales se puede deber a la corta vida de los estudios universitarios en Marketing que, como apuntábamos al inicio de este artículo, se remontan tan solo al año 2010 , frente a los estudios de empresa que en algunas universidades superan ya los 150 años de existencia. La consolidación de estos estudios, ya verificados en muchas facultades tras cinco años de existencia cumpliendo todos los parámetros exigidos por las autoridades evaluadoras, junto con la amplia demanda por parte del alumnado (el número de plazas solicitadas suele superar a las ofertadas en la mayoría de los centros) son datos objetivos que deberían animar a las editoriales a plantearse dotar de materiales nuevos a estos alumnos que en su futuro, sin duda, deberán utilizar el inglés específico de su campo mucho más que en otros entornos laborales.

\section{Bibliografía}

Barsanti, Ma Jesús (2000): "Relaciones entre el alemán y el francés en los léxicos específicos de Marketing, publicidad y comunicación”, en Casal, M. L., G. Conde, J. Lago, L. Pino, N. Rodríguez (coords.), La Lingüistica francesa en España camino del siglo XXI, Madrid, Arrecife, 119-124.

Blesa, Andreu e Inmaculada Fortanet (2003): "Análisis del artículo de investigación de marketing. Características propias", Ibérica, 5, 69-86.

Bobáková, Hana (2010): “Anglizismen im Marketingbereich”, Revista de Filología Alemana, Anejo II, 31-43.

Braga, Gloria y José Luis Belver (2016): "El análisis de libros de texto: una estrategia metodológica en la formación de los profesionales de la educación", Revista Complutense de Educación, 27(1), 199-218.

Bravo, Rafael, Julio Jiménez y José Miguel Pina (2011): "La docencia universitaria en inglés. Una aplicación al Marketing", en Paricio, J., M. C. Allueva, A. Lacruz y F. Cruz (coords.), 
Experiencias de innovación e investigación educativa en el nuevo contexto universitario, Zaragoza, Prensas Universitarias de Zaragoza, 545-562.

Bueno, Concepción y Magaly Hernández (2002): "Inglés con fines específicos: entonces y ahora", Humanidades Médicas, 2.1, 0-0. http://scielo.sld.cu/scielo.php?script=sci_arttextypid=S1727-8120200200010 0003 [Consulta: 8 septiembre 2016]

Dudley-Evans, Tony y Maggie Jo St. John (1998): Developments in English for Specific Purposes. A Multi-Disciplinary Approach, Cambridge, Cambridge University Press.

Durán, Ramiro (2000): "Análisis estadístico de la presencia de la lengua inglesa en la publicidad comercial española”, Aula: Revista de Pedagogía de la Universidad de Salamanca, 12, 87-101.

Durán, Ramiro (2002): La lengua inglesa en la publicidad española, Salamanca, Universidad de Salamanca.

Felices, Ángel M. (2005): "La teoría y la práctica en el desarrollo de la lengua para fines específicos en E/LE: Revisión crítica de los logros de la última década", Quaderns de Filologia. Estudis Lingüístics, X, 81-97.

Figueroa, Beatriz (1999): "El Inglés como lengua predominante en un diccionario plurilingüe de marketing, publicidad y comunicación", Babel afial: Aspectos de filología inglesa y alemana, 8, 93-102.

Fresno, Mercedes (2012): Reestructuración de los estudios de lengua inglesa con fines específicos en las carreras técnicas en la universidad pública española: análisis detallado de un corpus de informantes: hipótesis, previsiones y propuesta, tesis doctoral, Universidad Complutense de Madrid.

García, Verónica (2015): “Análisis del vocabulario en libros de texto para la enseñanza AICLE en $5^{\circ}$ de Educación Primaria”, Campo abierto: Revista de educación, 34(1), 29-47.

Gea, Ma Luisa (2005): “Advertising books: a linguistic analysis of blurbs", Ibérica, 10, 41-62.

González, Rocío (2015): “Análisis holístico, diacrónico y multimodal de libros de texto de inglés como lengua extranjera: Una nueva forma de mejorar la comprensión", Foro de Educación, 13 (19), 343-356.

Guilloto, Fernando (1994): La Carrera de comercio en Cádiz. Del arte de la contabilidad a las ciencias empresariales, Cádiz, Servicio de Publicaciones de la Universidad de Cádiz.

Guiu, David (2015): "Relación entre el Marketing y la Publicidad (y diferencias entre ambos)", SOCIALetic. http://www.socialetic.com/relacion-entre-el-Marketing-y-la-publicidad-y-diferencias-entre-ambos.html [consulta: 14 de octubre 2016]

Hyland, Ken (1999): "Talking to Students: Metadiscourse in Introductory Coursebooks", English for Specific Purposes, Vol. 18, 1, 3-26.

Hurtado, Jorge (2016): "Fundamentos de Marketing Turístico", Boletín Científico de las Ciencias Económico Administrativas del ICEA, 2, 4.

Jiménez, Rosa $\mathrm{M}^{\mathrm{a}}$ (2003): "Libros de texto para la enseñanza precoz del inglés: estudio comparativo”, RAEL: Revista Electrónica de Lingüística Aplicada, 2, 237-250.

Kotler, Philip y Gary Armstrong (2003): Fundamentos de Marketing, Ciudad de Méjico, Pearson Educación.

Lee, Seung-eun y Mary A. Littrell (2006): "Marketing cultural products on the internet: targeting cultural creatives", Clothing and Textiles Research Journal, 24(1), 33-45.

Luján, Carmen Isabel (2010): "La presencia de la lengua inglesa en carteles publicitarios en las calles de Las Palmas de Gran Canaria", Miscelánea: A Journal of English and American Studies, 41, 13-39.

Martín, Lourdes (2002): Marketing político: arte y ciencia de la persuasión en democracia, Barcelona, Paidós. 
Martínez, Simón R. (2008): “Presentación”, en Llano, F. y J. Calvo (eds.), Hoy Es Marketing 08, Madrid, ESIC Editorial.

Mendoza, Henry X., María G. Mendoza-Ponce y Karen A. Mendoza (2015): "Estrategia metodológica para favorecer la interdisciplinariedad entre las asignaturas de Inglés y Marketing", Dominio de las Ciencias, 1, 31-46.

Núñez, Julia M., F. José Rey y Nuria Ceular (2011): “Aplicación de la metodología CLIL (Content and Language Integrated Learning) al ámbito del Marketing", Revista d'innovació docent universitària: RIDU, 3, 99-105.

Palmer, Juan Carlos (1999): "Netvertising and ESP: genre-based analysis of target advertisements and its application in the Business English classroom", Ibérica, 1, 39-54.

Pérez, Alfredo, Norma Melitina Nodarse y Ma Antonia Mons (2013): "Sistema de actividades para desarrollar la comunicación intercultural mediante la escritura en inglés", Edumecentro, 5(3), 143-156.

Pérez, Andrea y Ignacio A. Rodríguez del Bosque (2014): “Identidad, imagen y reputación de la empresa: integración de propuestas teóricas para una gestión exitosa", Cuadernos de Gestión, Vol. 14, 1, 97-126.

Puchmüller, Andrea B., Jaquelina Noriega y Ma Verónica Fattori (2010): "Libros de texto y lectura en inglés: un análisis de la década del '60”, Diálogos Pedagógicos, Vol. 8, 15, 69-84.

Robinson, Pauline (1991): ESP Today: A Practitioner's Guide, Londres, Prentice Hall International.

Real Academia Española (2014): Mercadotecnia, en Diccionario de la lengua española, 23 ed. http://dle.rae.es/?w=mercadotecnia [Consulta: 16 octubre 2016]

Real Academia Española y Asociación de Academias de la Lengua Española (2005): Diccionario panhispánico de dudas, Bogotá, Santillana Ediciones Generales.

Santesmases, Miguel (2014): Marketing, Madrid, Ediciones Pirámide.

Venegas, Ana y Silvia Ocaña (2016): "El inglés en la publicidad: entre el exceso, el papanatismo y la naturalidad”, Anuncios: Semanario de publicidad y Marketing, 1532, 36-27.

Vicente, Ma Azucena (2014): “Marketing ecológico. Oportunidades, políticas y retos”, en Cubillo, J. M. y A. Blanci (coords.), Estrategias de Marketing Sectorial, Madrid, ESIC, 119-158.

Vieira, Francisco G. D., Agnaldo K. Higuchi, Rosemeri Schneider y Patricia Soares (2013): "Marketing Social Corporativo: estado da arte e proposição de um esquema conceitual", Revista de Ciências da Administração, 15 (37), 37-51.

Zenone, Luiz Claúdio (2006): Marketing social, San Pablo, Thomson Learning.

\section{Anexo: Libros de texto analizados (orden alfabético)}

Adamson, Donald (1991): Starting English for Business, Cambridge, Cambridge University Press.

Andrews, R.G.H. (1990): Written English for Business. 2nd Level, Oxford, Oxford University Press.

Ardó, Zsuzsanna (1988): English for Practical Management, Oxford, Oxford University Press.

Ashley, A. (1991) (1984): A Handbook of Commercial Correspondence, Oxford, Oxford University Press.

Barber, Eric (2007): 50 Ways to improve your Business English using the Internet, Oxford, Heinle. 
Barry, Robert E. (1988): Business English for the 90's, Englewood Cliffs, Prentice Hall International.

Bater, Bernard y Gerald Lees (1990): Written English for Business. 3rd Level, Oxford, Oxford University Press.

Benn, Colin y Paul Dummet (1992a): Business First, Oxford, Heinemann.

Benn, Colin y Paul Dummet (1992b): A First Course in Business English, Oxford, Heinemann.

Bennie, Michael (2009): A Guide to Good Business Communication, Oxford, How to Books Ltd.

Beresford, Cynthia (1988) (1984): Business Communication, Oxford, Oxford University Press.

Bethell, George y Tricia Aspinall (2009) (2003): Test your Business Vocabulary in Use, Intermediate, Cambridge, Cambridge University Press.

Blundell, John A. y Nigel M.G. Middlemiss (1991a) (1981): Career. Prospects, Oxford, Oxford University Press.

Blundell, John A. y Nigel M.G. Middlemiss (1991b) (1982): Career. Developments, Oxford, Oxford University Press.

Brieger, Nick y Jeremy Comfort (1989): Early Business Contacts, Cambridge, Cambridge University Press.

Brieger, Nick y Simon Sweeney (1994): The Language of Business English, Bristol, Prentice Hall International.

Brieger, Nick, Jeremy Comfort, Steve Hughes y Chris West (1987): Business Contacts, Englewood Cliffs, Prentice Hall International.

Brown, Pearson y John Allison (1991): Business Partners, Hove, Language Teaching Publications.

Campbell, Barbara (2000): English for Business, Boston, Harrap Thomson English Language Teaching.

Carrier, Michael (1985): Business Reading Skills, Edimburgo, Nelson.

Carrier, Michael (1988): Business Circles, Edimburgo, Nelson.

Ceramella, Nick y Elisabeth Lee (2010) (2008): Cambridge English for the Media, Cambridge, Cambridge University Press.

Clarke, Simon (2003): In Company, Oxford, McMillan.

Comfort, Jeremy (2004) (1996): Effective Telephoning, Oxford, Oxford University Press.

Comfort, Jeremy y Nick Brieger (1992a): Marketing. Intermediate-advanced, Londres, Prentice Hall International.

Comfort, Jeremy y Nick Brieger (1992b): Marketing. Upper-intermediate, Londres, Prentice Hall International.

Comfort, Jeremy, Rod Revell y Chris Stott (1990) (1984): Business Reports in English, Cambridge, Cambridge University Press.

Cotton, David (1989a) (1980): International Business Topics, Edimburgo, Nelson.

Cotton, David (1989b) (1988): Keys to Management, Edimburgo, Nelson.

Cotton, David (1992) (1984): World of Business, Londres, Bell and Hyman.

Cotton, David y Sue Robbins (1998) (1993): Business Class, Edimburgo, Longman.

Cotton, David, David Falvey y Simon Kent (2000): Market Leader, Intermediate, Edimburgo, Longman.

Cotton, David, David Falvey y Simon Kent (2006) (2001): Market Leader, Upper-Intermediate, Harlow, Pearson-Longman.

Cotton, David, David Falvey y Simon Kent (2012) (2002): Market Leader. Pre-Intermediate, Harlow, Pearson-Longman. 
Danks, Stephen y Lynne Cross (1989): Assignments in Business Studies, Edimburgo, Nelson. Darragh, Glenn y Edward R. Rosset (2008) (1998): New Guide to Business Letters, Irun, Stanley.

Davies, David y Douglas Pickett (1990): Preparing for English for Commerce, Englewood Cliffs, Prentice Hall International.

Dubicka, Iwonna y Margaret O'Keeffe (2016) (2006): Market Leader. Advanced, Harlow, Pearson-Longman.

Farral, Cate y Marianne Lindsley (2008): Professional English in Use: Marketing, Cambridge, Cambridge University Press.

Fletcher, Mark y Roger Hargreaves (1983): Good for Business, Edimburgo, Nelson.

Flower, John (1990): Build your Business Vocabulary, Hove, Language Teaching Publications.

Fowles, Anthony, Margaret Miller y Jean Stoker (1995): Executive Decisions, Londres, Nelson. Geffner, Andrea B. (1998): Business English, Nueva York, Barron's.

Giménez Moreno, Rosa (ed.) (2010): Words for Working. Professional and Academic English for International Business and Economics, Valencia, Universidad de Valencia.

Goddard, Christopher (1994): Business Idioms International, Englewood Cliffs, Prentice Hall International.

Goodale, Malcolm (1991) (1987): The Language of Meetings, Hove, Language Teaching Publications.

Goodwin, Joy y Lyn Strutt (2005): Test your Business Vocabulary in Use. Advanced, Cambridge, Cambridge University Press.

Gore, Sylee (2015) (2007): English for Marketing and Advertising, Oxford, Oxford University Press.

Grant, David y Robert Mclarty (2001): Business Basics, Oxford, Oxford University Press.

Greenall, Simon (1986): Business Targets, Oxford, Heinemann.

Gutjahr, Lothar y Sean Mahoney (2011) (2009): English for Sales and Purchasing, Oxford, Oxford University Press.

Hanks, Patrick y Jim Corbett (1989) (1986): Business Listening Tasks, Cambridge, Cambridge University Press.

Herrera, Honesto y Michael White (1994): Dive into English for Business and Economics, Madrid, McGraw Hill.

Hollet, Vicky, Roger Carter, Liz Lyon y Emma Tanner (1991) (1989): In at the Deep End, Oxford, Oxford University Press.

Horner, David y Peter Strutt (1996): Words at Work, Cambridge, Cambridge University Press. Howard-Williams, Deirdre y Cynthia Herd (1992): Business Words, Oxford, Heinemann.

Huggett, Renée (1990): Business Case Studies, Cambridge, Cambridge University Press.

Ibbotson, Mark y Bryan Stephens (2010) (2006): Business Start-up, Cambridge, Cambridge University Press.

Irvine, Mark y Marion Cadman (2000) (1999): Commercially Speaking, Oxford, Oxford University Press.

Jones Macziola, Sarah y Greg White (2001) (1993): Getting Ahead, Cambridge, Cambridge University Press.

Jones, Leo (2009) (2001): Working in English, Cambridge, Cambridge University Press.

Jones, Leo y Richard Alexander (2000) (1989): New International Business English, Cambridge, Cambridge University Press.

Jordan, Robert R. y Frederick I. Nixson (1989) (1986): Language for Economics, Londres, Collins. 
Lees, Gerald (1983): Negotiate in English, Edimburgo, Nelson.

Littlejohn, Andrew (1990) (1988): Company to company, Cambridge, Cambridge University Press.

Lloid, Angela y Anne Preier (1996): Business Communication Games, Oxford, Oxford University Press.

Mackenzie, Ian (1997a): English for Business Studies. Intermediate-advanced, Cambridge, Cambridge University Press.

Mackenzie, Ian (1997b): Management and Marketing, Hove, Language Teaching Publications.

Mackenzie, Ian (2002) (1997): English for Business Studies. Upper intermediate, Cambridge, Cambridge University Press.

Mascull, Bill (1996): Key Words in Business, Londres, Collins.

Mascull, Bill (2006): Business Vocabulary in Use. Beginners, Cambridge, Cambridge University Press.

Mascull, Bill (2010a) (2002): Business Vocabulary in Use. Intermediate, Cambridge, Cambridge University Press.

Mascull, Bill (2010b): Business Vocabulary in Use. Advanced, Cambridge, Cambridge University Press.

Matthew, Candace (1987): Business Interactions, Englewood Cliffs, Prentice Hall International.

Mawer, Jenny (1992): Business Games, Hove, Language Teaching Publications.

McCarthy, Michael, Jeanne McCarten, David Clark y Rachel Clark (2009): Grammar for Business, Cambridge, Cambridge University Press.

McKellen, Joy Sylvia (1990) (1985): Test your Business English, Londres, Collins.

Mohr, Boudewijn (1978): The Language of International Trade in English, Englewood Cliffs, Prentice Hall International.

Montagu, John H. (1980a): Practical Business English. Book I, Bucks, Hulton Educational Publications.

Montagu, John H. (1980b): Practical Business English. Book II, Bucks, Hulton Educational Publications.

Montagu, John H. (1980c): Practical Business English. Book III, Bucks, Hulton Educational Publications.

Moore, C. J. y Judy West (1985): Enterprise One, Londres, Heinemann.

Morrison, John (1994): Business Language Practice, Hove, Language Teaching Publications.

Naterop, Bertha J., Erich Weis y Eva Haberfellner (1991) (1977): Business Letters for All, Oxford, Oxford University Press.

Naterop, Bertha J. y Rod Revell (1991) (1987): Telephoning in English, Oxford, Oxford University Press.

Naunton, Jon (2000): Head for Business, Oxford, Oxford University Press.

Naunton, Jon (2009a) (2005): Profile 1, Oxford, Oxford University Press.

Naunton, Jon (2009b) (2005): Profile 2, Oxford, Oxford University Press.

Naunton, Jon (2009c) (2005): Profile 3, Oxford, Oxford University Press.

Nikolaenko, E.B. (2008): Business English, Tomsk, Tomsk Polytechnic University Publishing House.

Norman, Susan (1991a) (1982): We mean Business, Harlow, Longman.

Norman, Susan (1991b) (1983): We're in Business, Harlow, Longman.

O'Driscoll, Nina (2010): Market Leader. Marketing, Edimburgo, Pearson.

Powell, Mark (1996): Business Matters, Hove, Language Teaching Publications. 
Radice, Francis (1982): English for International Trade, Edimburgo, Nelson.

Renucci, Claude (1997): Inglés para el Marketing y la Negociación, Paris, Larousse.

Riley, David (1995): Check your English Vocabulary, Londres, Collins.

Robinson, Nick (2010): Cambridge English for Marketing, Cambridge, Cambridge University Press.

Rodgers, Drew (1999) (1997): English for Business Negotiations, Cambridge, Cambridge University Press.

Rowlands, K.E. (1983): Management Assignment, Londres, Hodder \& Stoughton.

Rowlands, K.E. (1989) (1979): Management English, Londres, Hodder \& Stoughton.

Ryan, John y John Richards (1991): Business Studies Today, Cambridge, Cambridge University Press.

Sandler, P.L. y Chris Stott (1991) (1981): Manage with English, Oxford, Oxford University Press.

Scullion, Malachy (1987): Managing People, Londres, Hodder y Stoughton.

Speegle, Roger y William B. Giesecke (1983): Business World, Oxford, Oxford University Press.

Spiro, Greg (1985): Business Information, Londres, Edward Arnold.

St John, Maggie-Jo (1994): Advertising and the Promotion Industry, Englewood Cliffs, Prentice Hall International.

Stanton, Alan (1990): Written English for Business. 1st level, Oxford, Oxford University Press.

Strutt, Peter (1992): Business English Usage, Harlow: Longman.

Sweeny, Simon (1999) (1997): English for Business Communication, Cambridge, Cambridge University Press.

Tayfoor, Valerie (1989): Examining Business Studies, Edimburgo, Nelson.

Taylor, Ken (2009) (2006): 51 Ways to improve your Business English, Londres, Summer Town Publishing.

Taylor, Liz (2008) (2004): International Express, Oxford, Oxford University Press.

Tullis, Graham y Tonya Trappe (2004): New Insights into Business, Edimburgo, Pearson.

Über Grosse, Christine y Robert E. Grosse (1988): Case Studies in International English, Englewood Cliffs, Prentice Hall International.

Watson-Delestree, Anne y Jimmie Hill (1998): The Working Week, Hove, Language Teaching Publications.

Whitcomb, Alan (1987a) (1980): Comprehensive Commerce, Edinburgo, Nelson.

Whitcomb, Alan (1987b) (1983): Office Practice, Edinburgo, Nelson.

White, Graham y Marjorie Khidhayir (1984) (1983): In Business, Edinburgo, Nelson.

White, Graham y Susan Drake (1996) (1989): Business Initiatives, Edinburgo, Logman.

Wilberg, Peter y Michael Lewis (1991) (1990): Business English, Londres, Language Teaching Publications.

Wilson, Martin (1987): Writing for Business, Edinburgo, Nelson.

Wyatt, Rawdon (2007): Check your Vocabulary for Business and Administration, Londres, AyC Black. 